TITLE:

\title{
Proper learning algorithm for functions of $\$ k \$$ terms under smooth distributions
}

\section{$\operatorname{AUTHOR(S):~}$}

Sakai, Yoshifumi; Takimoto, Eiji; Maruoka, Akira

\section{CITATION:}

Sakai, Yoshifumi ... [et al]. Proper learning algorithm for functions of $\$ k \$$ terms under smooth distributions. 数理解析研究所講究録 1995, 906: 236-243

ISSUE DATE:

1995-04

URL:

http://hdl.handle.net/2433/59438

RIGHT: 


\title{
Proper learning algorithm for functions of $k$ terms under smooth distributions
}

\author{
Yoshifumi Sakai Eiji Takimoto Akira Maruoka \\ Graduate School of Information Sciences, Tohoku University, Sendai 980-77, Japan \\ Email: $\{$ yoshif, t2, maruoka $\} @$ ecei.tohoku.ac.jp
}

\begin{abstract}
Summary: In this paper, we deal with a class written as $\mathcal{F}_{1} \circ \mathcal{F}_{2}{ }^{k}=\left\{g\left(f_{1}(v), \ldots, f_{k}(v)\right) \mid\right.$ $\left.g \in \mathcal{F}_{1}, f_{1}, \ldots, f_{k} \in \mathcal{F}_{2}\right\}$ for classes $\mathcal{F}_{1}$ and $\mathcal{F}_{2}$ characterized by "simple" descriptions and study the learnability of $\mathcal{F}_{1} \circ \mathcal{F}_{2}{ }^{k}$ from examples, where $\mathcal{F}_{1}$ and $\mathcal{F}_{2}$ are the classes of functions from $\Sigma^{k}$ to $\Sigma$ and those from $\Sigma^{n}$ to $\Sigma$, where $\Sigma=\{0,1\}$. Even if both of $\mathcal{F}_{1}$ and $\mathcal{F}_{2}$ are learnable, it is hard to learn $\mathcal{F}_{1} \circ \mathcal{F}_{2}{ }^{k}$ in general. For example, in the distribution free setting, it is known to be NP-hard to learn properly $k$-term DNF, which is represented as $\{\mathrm{OR}\} \circ \mathcal{T}_{n}{ }^{k}$, where $\mathcal{T}_{n}$ is the class of all monomials of $n$ variables. In this paper, we first introduce a probabilistic distribution, called a smooth distribution, which is a generalization of $q$-bounded distribution and product distribution, and define the learnability under this distribution. Then, we give an algorithm that properly learns $\mathcal{F}_{k} \circ \mathcal{T}_{n}{ }^{k}$ under smooth distribution in polynomial time for constant $k$, where $\mathcal{F}_{k}$ is the class of all Boolean functions of $k$ variables. The class $\mathcal{F}_{k} \circ \mathcal{T}_{n}{ }^{k}$ is called the functions of $k$ terms and although it was shown by Blum and Singh to be learned using DNF as a hypothesis class, it remains open whether it is properly learnable under distribution free setting.
\end{abstract}

\section{Introduction}

Since Valiant introduced PAC learning model [4], much effort has been devoted to characterize learnable classes of concepts on this model. Among such classes are the ones represented by some restricted Boolean formulas such as DNF, CNF, $k$-DNF, $k$-CNF, $k$-term DNF and $k$-clause CNF as well as the ones given by describing Boolean functions such as threshold functions. In each cases, the class is somehow defined by a "simple" description. In this paper, we deal with a class written as $\mathcal{F}_{1} \circ \mathcal{F}_{2}{ }^{k}=\left\{g\left(f_{1}(v), \ldots, f_{k}(v)\right) \mid\right.$ $\left.g \in \mathcal{F}_{1}, f_{1}, \ldots, f_{k} \in \mathcal{F}_{2}\right\}$ for classes $\mathcal{F}_{1}$ and $\mathcal{F}_{2}$ characterized by "simple" descriptions and study the learnability of $\mathcal{F}_{1} \circ \mathcal{F}_{2}{ }^{k}$ from examples, where $\mathcal{F}_{1}$ and $\mathcal{F}_{2}$ are the classes of functions from $\Sigma^{k}$ to $\Sigma$ and those from $\Sigma^{n}$ to $\Sigma$, where $\Sigma=\{0,1\}$. When the target function to be learned is $g\left(f_{1}(v), \ldots, f_{k}(v)\right)$ in $\mathcal{F}_{1} \circ \mathcal{F}_{2}{ }^{k}$ and both of $g$ and $f_{1}, \ldots, f_{k}$ are unknown, in general it is impossible to determine the values of $f_{1}(v), \ldots, f_{k}(v)$ even if pairs $\left(v, g\left(f_{1}(v), \ldots, f_{k}(v)\right)\right)$ are given as examples for sufficiently many $v$ 's in $\Sigma^{n}$. Hence, even if both of $\mathcal{F}_{1}$ and $\mathcal{F}_{2}$ are learnable, it is hard to learn $\mathcal{F}_{1} \circ \mathcal{F}_{2}{ }^{k}$ in general. For example, in the distribution free setting, it is NP-hard to learn properly $k$-term DNF, which is represented as $\{\mathrm{OR}\} \circ \mathcal{T}_{n}{ }^{k}$, where $\mathcal{T}_{n}$ is the class of all monomials of $n$ variables $[2,3]$.

Blum and Singh [1] studied the learnability of the class $\mathcal{F}_{k} \circ \mathcal{T}_{n}{ }^{k}$, denoted $\mathcal{F}_{k \text {-term }}$, where $\mathcal{F}_{k}$ is the class of all Boolean functions of $k$ variables, and showed that, for constant $k, \mathcal{F}_{k \text { term }}$ is learnable by hypothesis class $O\left(n^{k+1}\right)$-term DNF in the distribution free setting. Furthermore, they showed that, for any symmetric function $g$ other than AND, NAND, TRUE, and FALSE, proper learning $\{g\} \circ \mathcal{T}_{n}{ }^{k}$ is NP-hard.

In this paper, we first introduce a probabilistic distribution, called a smooth distribution, which is a generalization of $q$-bounded distribution and product distribution, and define the learnability under this distribution. Then, we give an algorithm that properly learns $\mathcal{F}_{k_{-} \text {term }}$ under smooth distribution in polynomial time for constant $k$. 


\section{Preliminaries}

In this extended abstract we follow the standard terminologies in PAC learning model unless otherwise stated. Obtaining positive and negative examples of a target function $f$ through oracles POS() and NEG(), a learning algorithm is expected to produce a hypothesis $h$ that approximates the target function $f$. A target function $f$ and a hypothesis $h$ are assumed to be Boolean functions of variables $x_{1}, \ldots, x_{n}$.

In the following, we often identify a Boolean formula with the Boolean function that it represents. So we regard the class of Boolean formulas as the corresponding class of Boolean functions. For a given Boolean formula (or the corresponding Boolean function) $f$, let $\mathcal{D}_{f}$ denote the set of all pairs $\left(D^{+}, D^{-}\right)$ of probability distribution $D^{+}$on the set of all positive examples of $f$ and probability distribution $D^{-}$ on the set of all negative examples of $f$. For a class $\mathcal{F}$ of Boolean formulas (or the corresponding class of Boolean functions), let $\mathcal{D}_{\mathcal{F}}$ denote $\bigcup_{f \in \mathcal{F}} \mathcal{D}_{f}$. Oracles generate examples independently according to some probability distributions $D^{+}$and $D^{-}$for some $\left(D^{+}, D^{-}\right)$in $\mathcal{D}_{f}$. In PAC learning model, the examples are usually assumed to be generated according to either an arbitrary distribution or a uniform distribution. In this paper we assume more general setting where the class of distributions according to which examples are drawn is taken arbitrarily as in Definition 2 below. Let $\Sigma=\{0,1\}$ and let $D$ be a distribution on subset $V$ of $\Sigma^{n}$. For a vector $v$ in $\Sigma^{n}$ and a subset $V^{\prime} \subseteq \Sigma^{n}$, let $D(v)$ denote the probability assigned to $v$ under $D$ and $D\left(V^{\prime}\right)$ denote $\sum_{v \in V^{\prime} \cap V} D(v)$. A Boolean function (formula) $g$ also represents the set of vectors $v$ in $\Sigma^{n}$ such that $g(v)=1$. So $D(g)$ represents $\sum_{f(g)=1} D(v)$ and $g \subseteq g^{\prime}$ means $\{v \mid g(v)=1\} \subseteq\left\{v \mid g^{\prime}(v)=1\right\}$. For Boolean functions $g$ and $g^{\prime}, D\left(g \mid g^{\prime}\right)$ denotes $D\left(g \wedge g^{\prime}\right) / D\left(g^{\prime}\right)$. The size of a Boolean function $g$ is the number of symbols appearing in the shortest description of $g$ under some reasonable encoding. Given a class of Boolean functions $\mathcal{F}, \mathcal{F}_{n, s}$ denotes the set of Boolean functions of $n$ variables with size at most $s$ in $\mathcal{F}$.

Definition 1 Let $f$ be a Boolean function, and let $\left(D^{+}, D^{-}\right) \in \mathcal{D}_{f}$. A Boolean function h $\varepsilon$-approximates $f$ under $\left(D^{+}, D^{-}\right)$if $D^{+}(f-h)<\varepsilon$ and $D^{-}(h-f)<\varepsilon$ hold.

Definition 2 Let $\mathcal{F}$ be a class of Boolean functions, and let $\mathcal{D}$ be a subset of $\mathcal{D}_{\mathcal{F}}$. An algorithm L learns $\mathcal{F}$ under $\mathcal{D}$ if and only if for any positive integers $n, s$, any target function $f$ in $\mathcal{F}_{n, s}$, any real numbers $\varepsilon, \delta$ with $0<\varepsilon, \delta<1$, and any pair of probability distributions $\left(D^{+}, D^{-}\right)$in $\mathcal{D} \cap \mathcal{D}_{f}$, when $L$ is given as input $n, s, \varepsilon$ and $\delta$ as well as access to $\operatorname{POS}()$ and $\mathrm{NEG}()$ that generate positive and negative examples independently according to $D^{+}$and $D^{-}$, respectively, $L$ halts in steps at most some polynomial in $n$, $s, 1 / \varepsilon$ and $1 / \delta$, and outputs a hypothesis $h$ in $\mathcal{F}_{n}$ that, with probability at least $1-\delta$, $\varepsilon$-approximates $f$ under $\left(D^{+}, D^{-}\right)$. Furthermore, if there exists a learning algorithm for $F$ under $\mathcal{D}$, then $F$ is called learnable under $\mathcal{D}$.

For a vector $v$ in $\Sigma^{n}$ and an integer $1 \leq i \leq n$, let $v_{i}$ denote the $i$ th component of $v$. For a vector $v$, let $\operatorname{true}(v)$ and false $(v)$ denote $\left\{i \mid v_{i}=1\right\}$ and $\left\{i \mid v_{i}=0\right\}$, respectively. Let $0^{n}$ and $1^{n}$ denote vectors $(0,0, \ldots, 0)$ and $(1,1, \ldots, 1)$ in $\Sigma^{n}$, respectively. For $v$ and $v^{\prime}$ in $\Sigma^{n}$, let $v \leq v^{\prime}$ denote the condition that $v_{i} \leq v_{i}^{\prime}$ for any $1 \leq i \leq n$, and let $v<v^{\prime}$ denote the condition that $v \leq v^{\prime}$ and $v \neq v^{\prime}$. For any subset $V$ of $\Sigma^{n}$, let $M i n_{\leq} V$ denote a subset of $V$ defined as

$$
\operatorname{Min}_{\leq} V=\left\{v \in V \mid \forall v^{\prime} \in V-\{v\} \quad v^{\prime} \not \leq v\right\}
$$

and let $\operatorname{Mon}(V)$ denote a monotone Boolean function of $n$ variables defined as

$$
\operatorname{Mon}(V)(v)= \begin{cases}1 & \exists v^{\prime} \in V \quad v^{\prime} \leq v \\ 0 & \text { otherwise. }\end{cases}
$$

Let $X_{n}$ denote the set of Boolean variables $x_{1}, \ldots, x_{n}$. Let $Y_{n}$ denote a set $X_{n} \cup\left\{\neg x_{i} \mid x_{i} \in X_{n}\right\}$. Let $\mathcal{F}_{n}$ denote the set of all Boolean functions of $n$ variables. Let TRUE and FALSE denote constant functions that take 1 and 0 , respectively. A conjunction of literals is called a term. Let $\mathcal{T}_{n}$ denote the set of all terms of literals $Y_{n}$. For a positive integer $k, \mathcal{T}_{n, \leq k}$ denote the set of terms $t$ of $n$ variables with 
$|l i t(t)| \leq k$. For a term $t, \operatorname{lit}(t)$ denotes the set of literals that appear in $t$. For any vector $v$ in $\Sigma^{n}, \sigma_{v}$ and $\tau_{v}$ denote terms of $n$ variables defined as

$$
\begin{aligned}
\sigma_{v} & =\bigwedge_{i \in \operatorname{true}(v)} x_{i} \wedge \bigwedge_{i \in \operatorname{false}(v)} \neg x_{i}, \\
\tau_{v} & =\bigwedge_{i \in \operatorname{true}(v)} x_{i} \quad\left(\text { e.g., }, \quad \tau_{0^{n}}=\mathrm{TRUE}\right)
\end{aligned}
$$

respectively.

For a Boolean function $g$ of $k$ variables and $k$-tuple $T=\left(t_{1}, \ldots, t_{k}\right)$ of terms of $n$ variables, $g(T)$ denotes a Boolean function of $n$ variables that takes value $g\left(t_{1}(v), \ldots, t_{k}(v)\right)$ for a vector $v$ in $\Sigma^{n}$. A Boolean function that can be represented as $g(T)$ for some $g$ in $\mathcal{F}_{k}$ and for some $T=\left(t_{1}, \ldots, t_{k}\right)$ in $\mathcal{T}_{n}{ }^{k}$ is called a function of $k$ terms, and $\mathcal{F}_{k_{-} \text {term }}$ denotes the class of functions of $k$ terms. For example, the class $\mathcal{F}_{2 \_ \text {term }}$ includes the function $\left(x_{1} \wedge \neg x_{2}\right) \oplus\left(x_{3} \wedge x_{4} \wedge x_{5}\right)$, where $\oplus$ denotes the exclusive OR function. A function $g(T)$ in $\mathcal{F}_{k_{-} \text {term }}$ can be represented as the composed function $g \circ T$ of function $g$ from $\Sigma^{k}$ to $\Sigma$ and function $T$ from $\Sigma^{n}$ to $\Sigma^{k}$. Similarly, in the following, we use notations such as $\sigma_{v}(T)$, $\tau_{v}(T), \sigma_{v} \circ T$ and $\tau_{v} \circ T$.

Definition 3 For positive integer $n$ and real number $0<p \leq 1$, probability distribution $D$ on $\Sigma^{n}$ is $p$ smooth if, for any vectors $v$ and $v^{\prime}$ in $\Sigma^{n}$ with Hamming distance $1, D(v) / D\left(v^{\prime}\right) \geq p$ holds . For a Boolean function $f$ of $n$ variables and real number $0<p \leq 1$, a pair of probability distributions $\left(D^{+}, D^{-}\right)$in $\mathcal{D}_{f}$ is p-smooth if there exists a p-smooth probability distribution $D$ on $\Sigma^{n}$ such that $D^{+}(v)=D(v) / D(f)$ for any positive vector $v$ of $f$, and $D^{-}(v)=D(v) / D(\neg f)$ for any negative vector $v$ of $f$. Let $\mathcal{S}_{f, p}$ denote the class of all $p$-smooth pairs $\left(D^{+}, D^{-}\right)$of $\mathcal{D}_{f}$. Furthermore, for a class $\mathcal{F}$ of Boolean functions, let $\mathcal{S}_{\mathcal{F}, p}$ denote the class $\bigcup_{f \in \mathcal{F}} \mathcal{S}_{f, p}$, and $\mathcal{S}_{\mathcal{F}, p}$ is simply written as $\mathcal{S}_{p}$ when no confusion arises.

\section{Learning algorithm}

A learning algorithm is assumed to get information about a target function $g \circ T$ through positive and negative examples of $g \circ T$. But, in general, it is impossible to know the value of $T(v)$ by observing the examples of $g \circ T$. To overcome the difficulty, the learning algorithm presented in this paper finds an $\varepsilon$-approximation of $g \circ T$ as follows. Instead of trying to find $T$, the algorithm seeks for a $k$-tuple of terms, denoted $\tilde{T}_{W, g, T}$, which can be found by observing sufficiently many examples of $g \circ T$. The $k$-tuple $\tilde{T}_{W, g, T}$ is determined by $W \subseteq \Sigma^{k}, g \in \mathcal{F}_{k}$, and $T=\left(t_{1}, \ldots, t_{k}\right) \in \mathcal{T}_{n}{ }^{k}$. As Lemma 2 states, it turns out that there exists a function, denoted $\tilde{g}_{W, g}$, in $\mathcal{F}_{k}$ such that $\tilde{g}_{W, g} \circ \tilde{T}_{W, g, T} \varepsilon$-approximates $g \circ T$. The fact that function $\tilde{g}_{W, g}$, which takes the same value as $g$ on $W$ (Proposition 1), is represented as the exclusive OR of at most $(k+1)$ monotone Boolean functions, guarantees that the learning algorithm can find $\tilde{T}_{W, g, T}$ in feasible time. Actually, the learning algorithm finds $\tilde{g}_{W, g} \circ \tilde{T}_{W, g, T}$ that $\varepsilon / 2$-approximates $g \circ T$. In the following, since $g, T$ and smooth distribution $\left(D^{+}, D^{-}\right)$are assumed to be fixed arbitrarily, we may drop suffices such as $g, T$ and $\left(D^{+}, D^{-}\right)$, e.g., $\tilde{g}_{W, g}$ and $\tilde{T}_{W, g, T}$ are simply written as $\tilde{g}_{W}$ and $\tilde{T}_{W}$, respectively. The learning algorithm first finds a set $\hat{\mathcal{U}}^{k}$ of $k$-tuples of terms that includes $\tilde{T}_{W}$ for appropriate $W$ such that $\tilde{g}_{W} \circ \tilde{T}_{W} \varepsilon / 2$-approximates $g \circ T$, and then finds $g^{\prime}$ in $\mathcal{F}_{k}$ and $U$ in $\hat{\mathcal{U}}^{k}$ by exhaustive search such that $g^{\prime} \circ U$ approximates $g \circ T$ with sufficient accuracy.

In this section, we first define $\tilde{g}_{W}$ and $\tilde{T}_{W}$ mentioned above, and then explain how the algorithm finds these functions.

A Boolean function $g$ in $\mathcal{F}_{k}, k$-tuple $T=\left(t_{1}, \ldots, t_{k}\right)$ in $\mathcal{T}_{n}{ }^{k}$ and $p$-smooth distribution $\left(D^{+}, D^{-}\right)$ in $\mathcal{D}_{g \circ T}$ are assumed to be fixed arbitrarily. Let $W$ be any subset of $\Sigma^{k}$. Let subsets $M_{W, 0}, M_{W, 1}, \ldots, M_{W, k+1}$ of $\Sigma^{k}$ be defined as

$$
M_{W, 0}=\left\{0^{k}\right\}
$$


and for $1 \leq l \leq k+1$

$$
M_{W, l}=\operatorname{Min}_{\leq}\left\{\begin{array}{l|l}
w^{\prime} \in W & \begin{array}{l}
\exists w \in M_{W, l-1} \\
w<w^{\prime}, g(w) \neq g\left(w^{\prime}\right)
\end{array}
\end{array}\right\} .
$$

Furthermore, let $d_{W, l}$ be defined to be $\operatorname{Mon}\left(M_{W, l}\right)$ for $0 \leq l \leq k+1$. It is clear that there exists $1 \leq l^{\prime} \leq k+1$ such that TRUE $=d_{W, 0} \supsetneqq d_{W, 1} \supsetneqq \cdots \supsetneqq d_{W, l^{\prime}}=d_{W, l^{\prime}+1}=\cdots=d_{W, k+1}=$ FALSE, and hence, $W$ is partitioned into the blocks

$$
\left\{W \cap\left(d_{W, 0}-d_{W, 1}\right), W \cap\left(d_{W, 1}-d_{W, 2}\right), \ldots, W \cap\left(d_{W, l^{\prime}-1}-d_{W, l^{\prime}}\right)\right\} .
$$

Furthermore, by definitions, it is easy to see that $g$ takes the same value on each block and the opposite values on any neighboring blocks. Let $\tilde{g}_{W}$ denote the Boolean function of $k$ variables defined as

$$
\tilde{g}_{W}=g\left(0^{k}\right) \oplus \bigoplus_{1 \leq l \leq k} d_{W, l}
$$

Then since, for any $0 \leq j \leq l^{\prime}-1$ and any vector $w$ in $W \cap\left(d_{W, j}-d_{W, j+1}\right)$,

$$
\tilde{g}_{W}(w)=g\left(0^{k}\right) \oplus \bigoplus_{1 \leq l \leq j} d_{W, l}(w)=g\left(0^{k}\right) \oplus \overbrace{1 \oplus \cdots \oplus 1}^{j}=g(w),
$$

the following proposition holds.

Proposition 1 For any vector $w$ in $W, g(w)=\tilde{g}_{W}(w)$.

Let $\operatorname{sign}_{g}$ denote the function defined as $\operatorname{sign}_{g}(j)=g\left(0^{k}\right) \oplus \overbrace{1 \oplus \cdots \oplus 1}^{j}$ for $1 \leq j \leq k$. Then $\operatorname{sign} n_{g}(j)$ represents the value that $g$ takes on the region $W \cap\left(d_{W, j}-d_{W, j+1}\right)$.

Let $M_{W}$ denote $\bigcup_{1 \leq i \leq k} M_{W, i}$. For $1 \leq i \leq k, \tilde{t}_{W, i}$ denotes a term defined as

$$
\tilde{t}_{W, i}=\bigwedge_{y \in Y} y, \quad \text { where } Y=\bigcap_{\substack{w \in M_{W} \\ w_{i}=1}} \operatorname{lit}\left(\tau_{w}(T)\right) .
$$

In the above definition, $\tilde{t}_{W, i}$ denotes FALSE when $w_{i}=0$ for any vector $w$ in $M_{W}$. Let

$$
\tilde{T}_{W}=\left(\tilde{t}_{W, 1}, \ldots, \tilde{t}_{W, k}\right) .
$$

Proposition 2 For any vector $w$ in $M_{W}, \tau_{w}(T)=\tau_{u}\left(\tilde{T}_{W}\right)$.

Proof: It suffices to show that $\operatorname{lit}\left(\tau_{w v}(T)\right)=\operatorname{lit}\left(\tau_{w}\left(\tilde{T}_{W}\right)\right)$. Recalling $T=\left(t_{1}, \ldots, t_{k}\right)$, we have $\tau_{w}(T)=\bigwedge_{w_{i}=1} t_{i}$. Since $\operatorname{lit}\left(t_{i}\right) \subseteq \operatorname{lit}\left(\tau_{w^{\prime}}(T)\right)$ holds for any $1 \leq i \leq k$ and any $w^{\prime}$ in $\Sigma^{k}$ with $w_{i}^{\prime}=1$, we have $\operatorname{lit}\left(t_{i}\right) \subseteq \operatorname{lit}\left(\tilde{t}_{W, i}\right)$, which implies $\operatorname{lit}\left(\tau_{w}(T)\right)=\bigcup_{w_{i}=1} \operatorname{lit}\left(t_{i}\right) \subseteq \bigcup_{w_{i}=1} \operatorname{lit}\left(\tilde{t}_{W, i}\right)=\operatorname{lit}\left(\tau_{w}\left(\tilde{T}_{W}\right)\right)$. On the other hand, since $w \in M_{W}$, we have $\operatorname{lit}\left(\tau_{w}(T)\right) \supseteq \bigcap_{w^{\prime} \in M_{W}, w_{i}^{\prime}=1} \operatorname{lit}\left(\tau_{w^{\prime}}(T)\right)=\operatorname{lit}\left(\tilde{t}_{W, i}\right)$ for any $i$ with $w_{i}=1$. Therefore, $\operatorname{lit}\left(\tau_{w^{\prime}}(T)\right) \supseteq \bigcup_{w_{i}=1} \operatorname{lit}\left(\tilde{t}_{W, i}\right)=\operatorname{lit}\left(\tau_{w^{\prime}}\left(\tilde{T}_{W}\right)\right)$.

Since $g$ and $\tilde{g}_{W}$ take the same value on $W, \tilde{g}_{W} \circ \tilde{T}_{W} \varepsilon$-approximates $g \circ T$ when $W$ mentioned above includes all vectors $w$ with $D^{g(w)}(\{v \mid T(v)=w\}) \geq \varepsilon / 2^{k}$ (Lemma 2), where $D^{1}$ and $D^{0}$ denote $D^{+}$ and $D^{-}$, respectively. In order to show this, we need to define some notations as follows. Let $\operatorname{rang} \epsilon(T)$ denote set $\left\{w \in \Sigma^{k} \mid \exists v \in \Sigma^{n} \quad w=T(v)\right\}$, and let $\operatorname{range}^{+}(T)=\operatorname{range}(T) \cup g$ and $\operatorname{range}^{-}(T)=$ range $(T) \cap(\neg g)$. Then range $(T)$ is partitioned into range ${ }^{+}(T)$ and range $-(T)$. Let range ${ }_{q}(T)$ denote the subset $\left\{w \in \operatorname{range}(T) \mid D^{g(w)}\left(\sigma_{w}(T)\right) \geq q\right\}$, where $D^{g(w)}\left(\sigma_{w}(T)\right)$ denotes $D^{g(w)}\left(\left\{v \in \Sigma^{n} \mid T(v)=w\right\}\right)$. Let range $\stackrel{\geq}{q}^{+}(T)={\text { range } \geq_{q}}(T) \cap g$ and range $-_{\geq_{q}}(T)=$ range $_{\geq_{q}}(T) \cap(\neg g)$. Then it is easy to see the following lemma. 
Lemma 1 If a Boolean function $h$ satisfies $(g \circ T)(v)=h(v)$ for any $w$ in range $e_{\geq \varepsilon / 2^{k}}(T)$ and any $v$ in $\Sigma^{n}$ with $T(v)=w$, then $h \varepsilon$-approximates $g \circ T$ under $\left(D^{+}, D^{-}\right)$.

Using Propositions 1, 2, and Lemma 1, we can show the following lemma.

Lemma 2 If range ${\text { I } / 2^{k}}(T) \subseteq W$, then $\tilde{g}_{W} \circ \tilde{T}_{W} \varepsilon$-approximates $g \circ T$ under $\left(D^{+}, D^{-}\right)$.

Proof: Let $w$ be any vector in $W$ and let $j$ be a suffix such that $w \in d_{W, j}-d_{W, j+1}$, that is, $d_{W, j}(w)=1$ and $d_{W, j+1}(w)=0$. Since $w \in W$, we have $g(w)=\tilde{g}_{W}(w)$ by Proposition 1 . Therefore, since $\tilde{g}_{W}$ takes the same value on $d_{W, j}-d_{W, j+1}$ and $w \in d_{W, j}-d_{W, j+1}$, we have $g(w)=\tilde{g}_{W}\left(w^{\prime}\right)$ for any $w^{\prime}$ in $d_{W, j}-d_{W, j+1}$.

Therefore, if $T(v)=w$ implies $\tilde{T}_{W}(v) \in d_{W, j}-d_{W, j+1}$, then $(g \circ T)(v)=\left(\tilde{g}_{W} \circ \tilde{T}_{W}\right)(v)$ for any $v$ in $\Sigma^{n}$

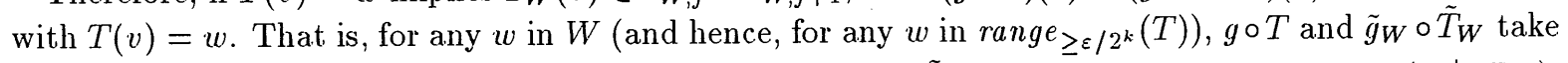
the same value on $\{v \mid T(v)=w\}$. Thus, by Lemma $1, \tilde{g}_{W} \circ \tilde{T}_{W} \varepsilon$-approximates $g \circ T$ under $\left(D^{+}, D^{-}\right)$. In the following, we show that $T(v)=w$ implies $\tilde{T}_{W}(v) \in d_{W, j}-d_{W, j+1}$.

Since $w$ in $\operatorname{Mon}\left(M_{W, j}\right)$, there exists $w^{\prime}$ in $M_{W, j}$ such that $w^{\prime} \leq w$. From Proposition 2, we have

$$
\tau_{w}(T) \subseteq \tau_{w^{\prime}}(T)=\tau_{w^{\prime}}\left(\tilde{T}_{W}\right) \subseteq \operatorname{Mon}\left(M_{W, j}\right) \circ \tilde{T}_{W}=d_{W, j} \circ \tilde{T}_{W}
$$

On the other hand,

$$
d_{W, j+1} \circ T=d_{W, j+1} \circ\left(t_{1}, \ldots, t_{k}\right) \supseteq d_{W, j+1} \circ\left(\tilde{t}_{W, 1}, \ldots, \tilde{t}_{W, k}\right)=d_{W, j+1} \circ \tilde{T}_{W}
$$

since, for any $1 \leq i \leq k, \operatorname{lit}\left(t_{i}\right) \subseteq \operatorname{lit}\left(\tilde{t}_{W, i}\right)$, that is, $t_{i} \supseteq \tilde{t}_{W, i}$. Therefore we have

$$
\begin{aligned}
T(v)=w & \Rightarrow\left(\tau_{w} \circ T\right)(v)=1 \text { and }\left(d_{W, j+1} \circ T\right)(v)=0 \\
& \Rightarrow\left(d_{W, j} \circ \tilde{T}_{W}\right)(v)=1 \text { and }\left(d_{W, j+1} \circ \tilde{T}_{W}\right)(v)=0 \\
& \Rightarrow\left(\left(d_{W, j}-d_{W, j+1}\right) \circ \tilde{T}_{W}\right)(v)=1 \\
& \Rightarrow \tilde{T}_{W}(v) \in\left(d_{W, j}-d_{W, j+1}\right)
\end{aligned}
$$

Let $f=g \circ T$ be a target function and let $W$ be any subset of $\Sigma^{k}$ such that range $\geq \varepsilon / 2^{k+1} \subseteq W$. Lemma 2 says that, in order to obtain $\tilde{T}_{W}=\left(\tilde{t}_{W, 1}, \ldots, \tilde{t}_{W, k}\right)$ such that $\tilde{g}_{W} \circ \tilde{T}_{W} \varepsilon / 2$-approximates $f$, it is sufficient to find $\tau_{w}(T)$ for each $w$ in $M_{W}$, because $\tilde{t}_{W, i}=\wedge\left(\bigcap_{w \in M_{W}, w_{i}=1} \operatorname{lit}\left(\tau_{w}(T)\right)\right)$.

To find $\tau_{w}(T)$ for each $w$ in $M_{W}$, the algorithm finds sets $\left\{\tau_{w}(T) \mid w \in M_{W, l}\right\}$ for $l=0,1, \ldots, k$, repeatedly. More precisely, to find $\tau_{w^{\prime}}(T)$ for each $w^{\prime}$ in $M_{W_{l} l}$, the algorithm uses $\tau_{w}(T)$ previously found for $w$ in $M_{W, l-1}$ with $w<w^{\prime}$. Since $w<w^{\prime}$ holds,

$$
\operatorname{lit}\left(\tau_{w^{\prime}}(T)\right)=\operatorname{lit}\left(\tau_{w^{\prime}}(T)\right) \cup \bigcup_{\substack{1 \leq i \leq k \\ w_{i}=0, w_{i}^{\prime}=1}} \operatorname{lit}\left(t_{i}\right) .
$$

In order to find $\tau_{w^{\prime}}(T)$, the algorithm tries to find a set $V$ consisting of sufficient number of vectors generated according to $D^{g\left(w^{\prime}\right)}$ with $\sigma_{w^{\prime}}(T)(v)=1$ (that is, $T(v)=w^{\prime}$ ), and to compute $\wedge\left\{y \in Y_{n}\right.$. $\forall v \in V \quad y(v)=1\}$. There is, however, no obvious way to know the value of $T(v)$ for vector $v$. So we explore conditions such that $T(v)=w^{\prime}$ holds for some $w^{\prime}$ satisfying the conditions mentioned above. The conditions have to be expressed in terms of $v$ and $\tau_{w^{\prime}}(T)$ without referring to $T(v)$. The conditions we notice consist of three conditions. The first condition is $\tau_{w}(T)(v)=1$. The second condition is the one that guarantees $t_{i}(v)=0$ for all $i$ with $w_{i}^{\prime}=0$. Provided that $y_{i}$ is chosen from $\operatorname{lit}\left(t_{i}\right)-\operatorname{lit}\left(\tau_{w^{\prime}}(T)\right.$ ) for each $i$ with $w_{i}^{\prime}=0$, let $r=\bigwedge_{i \in f a l s e\left(w^{\prime}\right)} \neg y_{i}$. The second condition we adopt is $r(v)=1$ for such $y_{i}$ 's which are found by exhaustive search. Then, if $v$ satisfies these two conditions, we can easily see that 
$w \leq T(v) \leq w^{\prime}$ holds. The third condition we take is $f(v)=g\left(w^{\prime}\right)$. When $w^{\prime}$ is the minimal vector among $w^{\prime \prime}$ in range $(T)$ such that $g\left(w^{\prime \prime}\right) \neq g(w)$ and that $w^{\prime \prime} \geq w$, it follows that $f(v)=g(T(v))=g\left(w^{\prime}\right)$ for $T(v) \geq w$ implies $T(v) \geq w^{\prime}$. Thus the third condition, together with the first and second conditions, guarantees that $T(v)=w^{\prime}$ (Lemma 3$)$.

Using these three conditions, the algorithm finds a set $V$ of sufficient number of $v$ 's such that $T(v)=w^{\prime}$ and computes set $\left\{y \in Y_{n} \mid \forall v \in V \quad y(v)=1\right\}$. Literals in $\left\{y \in Y_{n} \mid \forall v \in V \quad y(v)=1\right\}$ are candidates for literals corresponding to $\tau_{w^{\prime}}(T)$, i.e., those appearing in $\bigwedge_{i \in t r u e\left(w^{\prime}\right)} t_{i}$. Since there may be a literal $\neg y_{i}$ appearing in $r$ but not in $\bigwedge_{i \in \operatorname{true}\left(w^{\prime}\right)} t_{i}$, it is necessary to remove all such literals from $\left\{y \in Y_{n} \mid \forall v \in V \quad y(v)=1\right\}$ to obtain $\operatorname{lit}\left(\tau_{w^{\prime}}(T)\right)$. In algorithm LEARN given in Figure 1, a possible set of such literals is denoted by $\rho$.

The argument above suggests to take as $W$ the set, denoted $W$, which is defined as follows.

$$
\begin{aligned}
\hat{W}= & \left\{w \in \text { range }^{+}(T) \mid \exists w^{\prime} \in \text { range }_{\geq \varepsilon / 2^{k+1}}^{+}(T) \quad w \leq w^{\prime}\right\} \\
& \cup\left\{w \in \text { range }^{-}(T) \mid \exists w^{\prime} \in \text { range }_{\geq \varepsilon / 2^{k+1}}^{-}(T) \quad w \leq w^{\prime}\right\} .
\end{aligned}
$$

Let child $_{\hat{W}}(w)$ denote $\operatorname{Min}_{\leq}\left\{w^{\prime} \in \hat{W} \mid w^{\prime} \geq w, g\left(w^{\prime}\right) \neq g(w)\right\}$. Then clearly, for any $w^{\prime}$ in $M_{\hat{W}, l}$, there exists $w^{\prime}$ in child $_{\hat{W}}(w)$ such that $w \in M_{\hat{W}, l-1}$, where $1 \leq l \leq k$. Note that if $w^{\prime} \in \operatorname{child}_{\hat{W}}(w)$, then $\tau_{w}^{\prime}(T) \varsubsetneqq \tau_{w}(T)$ holds. Let $\mathcal{R}_{w}$ be defined as

$$
\mathcal{R}_{1^{k}}=\{\mathrm{TRUE}\}
$$

and for $w$ in $\Sigma^{k}-\left\{1^{k}\right\}$,

$$
\mathcal{R}_{w}=\left\{r \in \mathcal{T}_{n, \leq k} \mid r \neq \mathrm{FALSE}, r=\bigwedge_{i \in \text { false }(w)} \neg y_{i}, y_{i} \in \operatorname{lit}\left(t_{i}\right)-\operatorname{lit}\left(\tau_{w}(T)\right)\right\} .
$$

Then, we can show the following lemmas.

Lemma 3 For any vector $w$ in $M_{\hat{W}}$, any vector $w^{\prime}$ in child $\hat{W}_{W}(w)$ and any term $r$ in $\mathcal{R}_{w^{\prime}}$,

$$
\tau_{u w^{\prime}}(T) \wedge r=(g \circ T)^{g\left(w^{\prime}\right)} \wedge \tau_{w}(T) \wedge r
$$

holds, where $(g \circ T)^{1}$ and $(g \circ T)^{0}$ denotes $g \circ T$ and $\neg(g \circ T)$, respectively.

Note that the above lemma implies that $D^{g\left(w^{\prime}\right)}\left(\tau_{w}(T) \wedge r\right)=D^{g\left(w^{\prime}\right)}\left(\tau_{w^{\prime}}(T) \wedge r\right)$, and hence $D^{g\left(w^{\prime}\right)}(y \mid$ $\left.\tau_{w}(T) \wedge r\right)=1$ for any $y$ in $\operatorname{lit}\left(\tau_{w^{\prime}}(T) \wedge r\right)$.

Lemma 4 Let $\left(D^{+}, D^{-}\right) \in \mathcal{S}_{g \circ T, p^{\prime}}$. For any $w$ in $\dot{W}$, any $w^{\prime}$ in child $\hat{W}(w)$ and $r^{\circ}$ in $\mathcal{R}_{w^{\prime}}$,

$$
D^{g\left(w^{\prime}\right)}\left(\tau_{w}(T) \wedge r\right) \geq \beta
$$

holds, and for any $x_{i}$ with $\left\{x_{i}, \neg x_{i}\right\} \cap \operatorname{lit}\left(\tau_{w^{\prime}}(T) \wedge r\right)=\emptyset$,

$$
\gamma \leq D^{g\left(w^{\prime}\right)}\left(x_{i} \mid \tau_{w}(T) \wedge r\right) \leq 1-\gamma
$$

holds, where $\beta=\varepsilon p^{k} / 2^{2 k+1}$ and $\gamma=p / 2$.

We are now ready to construct Algorithm LEARN to learn $\mathcal{F}_{k} \circ \mathcal{T}_{n}{ }^{k}$ under $p$-smooth distributions. An outline of the algorithm is given as follows. Algorithm LEARN first obtains samples $S^{+}$of $m$ positive examples and $S^{-}$of $m$ negative examples by calling POS() and NEG() $m$ times, respectively, where $m$ is a sufficiently large number. Then, LEARN puts $\mathcal{U}_{0}=\{$ TRUE $\}$, and computes the sets $\mathcal{U}_{1}, \ldots, \mathcal{U}_{k}$ such that $\left\{\tau_{w}(T) \mid w \in M_{\hat{W}, l}\right\} \subseteq \mathcal{U}_{l}$ for $1 \leq l \leq k$, repeatedly. For $1 \leq l \leq k, \mathcal{U}_{l}$ is computed by using $\mathcal{U}_{l-1}$ as follows. Assume that LEARN has $\mathcal{U}_{l-1}$ such that $\left\{\tau_{w}(T) \mid w \in M_{\hat{W}, l-1}\right\} \subseteq \mathcal{U}_{l-1}$ holds, and 


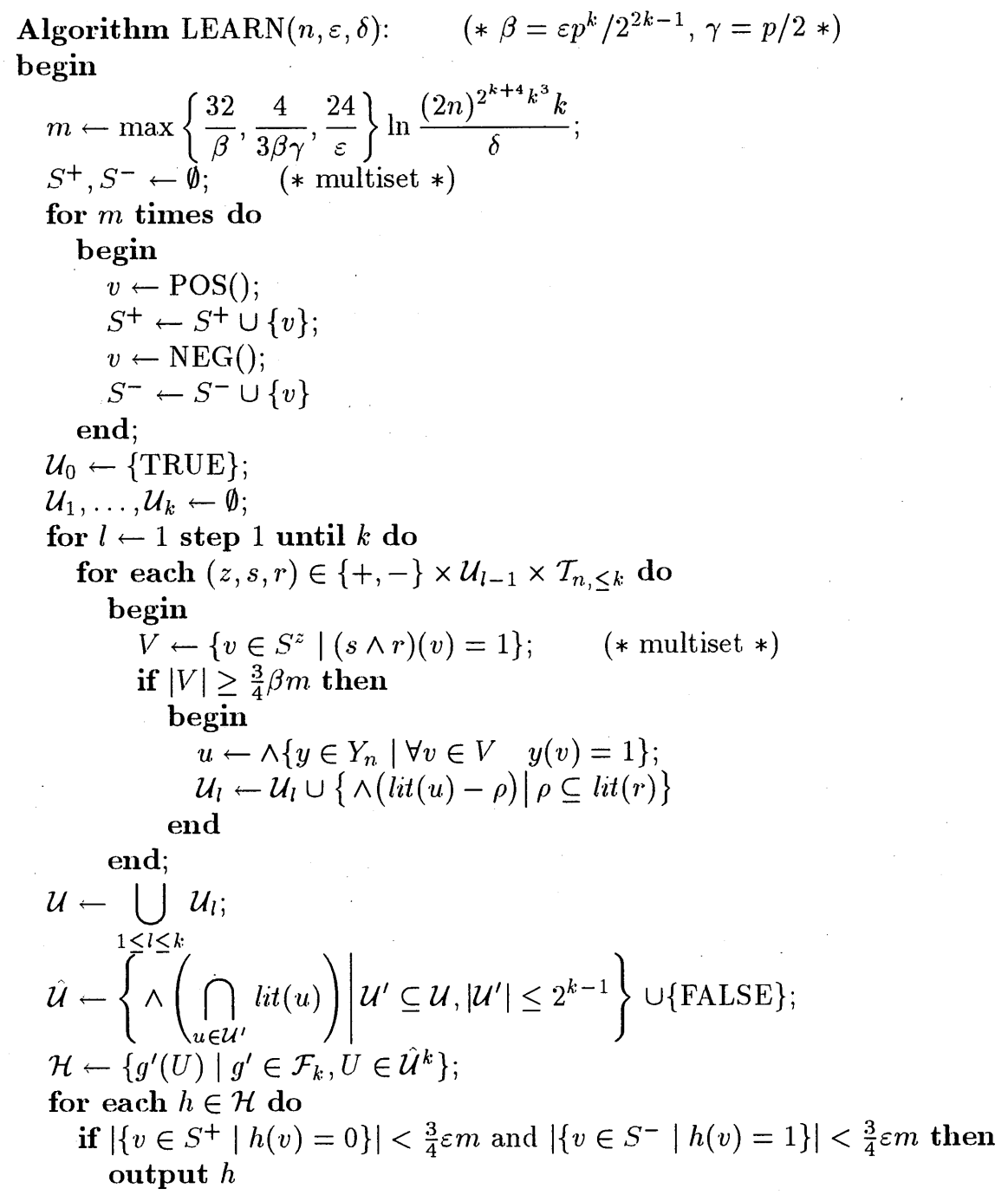
end.

Figure 1: Algorithm LEARN 
let $w^{\prime}$ be any vector in $M_{\hat{W}, l}$. There exists $w$ in $M_{\hat{W}, l-1}$ such that $w^{\prime} \in \operatorname{child}_{\hat{W}}(w)$. If the parameter $(z, s, r)$ of for sentence is $\left.\operatorname{sign}_{g}(l), \tau_{w}(T), r_{w^{\prime}}\right)$ for $r_{w^{\prime}} \in \mathcal{R}_{w^{\prime}}$, then, by Lemma 4 , the set $V$ of vectors $v$ in $S^{\text {sign }_{g}(l)}$ with $\left(\tau_{w^{\prime}}(T) \wedge r_{w^{\prime}}\right)(v)=1$ satisfies, with sufficiently high probability, $|V| \geq \frac{3}{4} \beta m$. Then, LEARN computes the set $\left\{y \in Y_{n} \mid \forall v \in V \quad y(v)=1\right\}$. Since by Lemma 4, for any literal $y$ not in $\operatorname{lit}\left(\tau_{w^{\prime}}(T) \wedge r_{w^{\prime}}\right)$, both of the probabilities of $y(v)=1$ and $y(v)=0$ are lower bounded by some constant (given as $\gamma=p / 2$ ) when $v$ is generated according to $D^{f\left(w^{\prime}\right)}$, a literal in $\left.\operatorname{lit}_{\left(w^{\prime}\right.}(T) \wedge r_{w^{\prime}}\right)$, with high probability, does not appear in $\left\{y \in Y_{n} \mid \forall v \in V \quad y(v)=1\right\}$ when $|V|$ is sufficiently large, which implies $\left\{y \in Y_{n} \mid \forall v \in V \quad y(v)=1\right\} \subseteq \operatorname{lit}\left(\tau_{w^{\prime}}(T) \wedge r_{w^{\prime}}\right)$ with high probability, and hence $\left\{y \in Y_{n} \mid \forall v \in V \quad y(v)=1\right\}=\operatorname{lit}\left(\tau_{w^{\prime}}(T) \wedge r_{u^{\prime}}\right)$. Putting $\rho$ a possible set of literals in lit $\left(r_{w^{\prime}}\right)$ but not in lit $\left(\tau_{w^{\prime}}(T)\right)$, LEARN produces $\wedge\left(\left\{y \in Y_{n} \mid \forall v \in V \quad y(v)=1\right\}-\rho\right)$ and adds it to $\mathcal{U}_{l}$. Therefore, since for sentence is executed for all the possible combinations of parameters $z, s, r$ in the sets given in the algorithm, we have that, with high probability, $\left\{\tau_{w^{\prime}}(T) \mid w^{\prime} \in M_{\hat{W}, l}\right\} \subseteq \mathcal{U}_{l}$ holds. Since we start with $\left\{\tau_{w}(T) \mid w \in M_{\hat{W}, 0}\right\}=\{$ TRUE $\}=\mathcal{U}_{0}$, it follows that $\left\{\tau_{w \prime}(T) \mid w^{\prime} \in M_{\hat{W}, l}\right\} \subseteq \mathcal{U}_{l}$ holds with high probability for $1 \leq l \leq k$. Let $\mathcal{U}=\bigcup_{1 \leq l \leq k} \mathcal{U}_{l}$. Then, since $\tilde{t}_{W, i}=\wedge\left(\bigcap_{w \in M_{W, w_{i}=1}} \operatorname{lit}\left(\tau_{w}(T)\right)\right)$ for $1 \leq i \leq k, \tilde{t}_{\hat{W}, i}$ is represented as $\wedge\left(\bigcap_{u \in \mathcal{U}^{\prime}}\right.$ lit $\left.(u)\right)$ for some appropriate set $\mathcal{U}^{\prime}$ of at most $2^{k-1}$ terms in $\mathcal{U}$. Let $\hat{\mathcal{U}}$ be the set of all possible terms $\wedge\left(\bigcap_{u \in \mathcal{U}^{\prime}}\right.$ lit $\left.(u)\right)$ for such $\mathcal{U}^{\prime}$ 's. Finally, LEARN obtains the desired hypothesis by checking all the combinations $g^{\prime}$ in $\mathcal{F}_{k}$ and $\left(\tilde{t}_{1}, \ldots, \tilde{t}_{k:}\right)$ in $\hat{\mathcal{U}}^{k}$ until $g^{\prime} \circ\left(\tilde{t}_{1}, \ldots, \tilde{t}_{k}\right)$ approximates $g \circ T$ with sufficient accuracy.

\section{Correctness}

The correctness of algorithm is verified by the following lemmas, which immediately implies Theorem 1 .

Lemma 5 With probability at least $1-\delta / 2, \mathcal{H}$ that Algorithm LEARN computes includes an $\varepsilon / 2$ approximation of $g \circ T$ in $\mathcal{F}_{k_{-} \text {term }}$ under $\left(D^{+}, D^{-}\right)$in $\mathcal{S}_{p}$.

Lemma 6 If $\mathcal{H}$ that Algorithm. LEARN computes includes an $\varepsilon / 2$-approximation of $g \circ T$ in $\mathcal{F}_{k \text { _term }}$ under $\left(D^{+}, D^{-}\right)$in $\mathcal{S}_{p}$, then LEARN outputs, with probability at least $1-\delta / 2$, h in $\mathcal{F}_{k_{-} \text {term }}$ that $\varepsilon-$ approximates $g \circ T$ under $\left(D^{+}, D^{-}\right)$.

Lemma 7 Algorithm LEARN halts in time $O\left(\left(n^{2^{k+4} k^{3}} / \varepsilon p^{k+1}\right) \ln (n / \delta)\right)$.

Theorem 1 If $k$ is constant and $p$ is bounded from below by the inverse of some polynomial in $n, \mathcal{F}_{k-t}$ term is learnable under $\mathcal{S}_{p}$.

\section{References}

[1] A. Blum, M. Singh, Learning functions of $k$ terms, in Proceedings of the 3rd Annual Workshop on Computational Learning Theory, Morgan Kaufmann, 1990, pp.144-153.

[2] M. Kearns, M. Li, L. Pitt and L. G. Valiant, On the Learnability of Boolean Formulae, In proceedings of the 19th Annual ACM Symposium on Theory of Computing, 1987, pp.285-295.

[3] L. Pitt and L. G. Valiant, Computational limitation on learning from examples, Journal of the ACM, Vol.35, No.4, 1988, pp.965-984.

[4] L. G. Valiant, A theory of the learnable, Communications of the ACM, 27(11), 1984, pp.1134-1142. 